\title{
A VISITA AO BRASIL DE FRANZ J, INGELFINGER E SUA PARTICIPAÇÃO NA DIVULGAÇÃO DOS CONHECIMENTOS SOBRE O MEGAESÓFAGO CHAGÁSICO
}

\author{
*J.M. de Rezende, U.G. Meneghelu
}

Faculdade de Medicina da Universidade Federal de Goiás; Faculdade de Medicina de Ribeirão Preto da Universidade de São Paulo e Sociedade Brasileira de História da Medicina

UnITERMOS: Doença de Chagas. Megaesôfago chagásico. Teste da metacolina.

KEY wORDs: Chagas Disease. Chagasic megaesophagus. Mecholyl teste.

Na década de 50, Franz Joseph Ingelfinger se destacava nos Estados Unidos como um dos maiores expoentes da gastroenterologia mundial, sendo admirado e respeitado por todos que o conheciam, como clínico, professor e pesquisador.

Natural da Alemanha, nascera na cidade de Dresden, em 1910, seus pais emigraram para os Estados Unidos quando tinha 12 anos de idade. Graduou-se em medicina na Universidade de Harvard, trabalhou algum tempo em Philadelphia e, aos 30 anos, assumiu a chefia do Departamento de Gastroenterologia do Evans Memorial Hospital, em Boston, onde permaneceu por 27 anos. Por sua marcante personalidade, competência e atuação, transformou o serviço sob sua direção em um Centro de excelência e escola de pós-graduação da especialidade. Ali se formaram dezenas de novos gastroenterologistas, que se tornaram líderes da nova geração tanto nos Estados Unidos como em outros países.

*Correspondência: $J$ offre Marcondes de Rezende Rua J oão de Abreu, 744 - Apto. 02 - S. 0 este Cep: 74120-110 - G oiânia - G 0

Fone: 251-3768 / Fax: (62) 251-3161 jmrezende@ mail.cultura.com.br
Após sua aposentadoria oficial, foi editor do New England Journal of Medicine, contribuindo com o seu tirocínio para manter o elevado conceito de que desfruta este periódico na comunidade médica.

Sua carreira foi pontuada de realizações bem sucedidas, até o seu falecimento em 1980, tendo recebido em vida o reconhecimento de várias instituições sob a forma de premiações, láureas e honrarias 5 .

Foi um dos pioneiros nos estudos da motilidade e registros manométricos do esôfago, da motilidade intestinal e absorção entérica, que eram as áreas de seu maior interesse.

Em 1948, Ingelfinger ${ }^{10}$ e, a seguir, em 1949 e 1951, Kramer e Ingelfinger ${ }^{19,20}$ descreveram o teste do Mecholyl ou metacolina na acalásia do esôfago e, em 1958, Ingelfinger publicou a mais completa revisão já feita até então sobre a fisiologia e fisiopatologia do esôfago, trabalho que se tornou clássico e fonte de referência obrigatória ${ }^{14}$.

O teste da metacolina, utilizado no diagnóstico diferencial da acalásia, baseia-se na hiper-reatividade das estruturas desnervadas ao estímulo farmacológico. Sendo a acalásia do esôfago uma afecção decorrente de dano da inervação intrínseca do esôfago, a muscu- latura deste órgão responde ao estímulo colinérgico com fortes contrações, que podem ser observadas à radioscopia ou inscritas em um sistema de registro manométrico.

Desde os trabalhos de Amorim e Correia Neto', Etzel ${ }^{6}$ e Correia Neto e Etzel ${ }^{4}$ que se conheciam, as lesões histopatológicas dos plexos intramurais do trato digestivo encontradas nos casos de megaesôfago e megacólon endêmicos, considerados ambos como manifestações de uma doença sistêmica.

Estudos clínicos ${ }^{25,26}$, sorológicos ${ }^{7,21}$ e anatomopatológicos ${ }^{15,24}$ posteriores indicavam como etiologia do megaesôfago e megacólon endêmicos que a doença de Chagas seria a responsável pelas lesões da inervação intrínseca descritas.

Koeberle, ${ }^{16-18}$ em estudos quantitativos de neurônios dos plexos intramurais, especialmente do plexo de Auerbach, tanto em casos humanos como em animais, demonstrou que a desnervação intrínseca produzida pela doença de Chagas ocorria com intensidade variável não somente no trato digestivo como em outros órgãos e sistemas, o que the permitiu conceituar a doença de Chagas como enfermidade do sistema nervoso autônomo. 
Com esta nova visão sobre a doença de Chagas e a comprovação da etiologia chagásica do megaesôfago e megacólon endêmicos, o teste da metacolina passou a ter um interesse especial como prova farmacológica de desnervação, não somente do esôfago como de outros segmentos do trato digestivo e outros órgãos eventualmente comprometidos pela doença de Chagas.

Os primeiros contatos com o Prof. Ingelfinger foram estabelecidos por um de nós (JMR), por correspondência, em abril de 1958, quando, por sugestão do Dr. Pedro Ribeiro de Carvalho, do Rio de Janeiro, foram-lhe enviadas separatas de alguns artigos publicados sobre o megaesôfago endêmico no Brasil e sua relação com a doença de Chagas.

Em carta de 22/4/58, o Prof. Ingelfinger acusava o recebimento das separatas e, referindo-se à relação entre megaesôfago e a doença de Chagas, declarou:

"This is a most fascinating correlation and it may provide us with a lead as to the origin of this mysterious disease."

Em 28/9/58, um de nós (JMR) enviouthe dois outros artigos publicados na Revista Goiana de Medicina, nos quais o megaesôfago era considerado como síndrome e não como doença. Em resposta datada de 21/10/1958, o Prof. Ingelfinger assim se manifestou:

"I am quite ready to be convinced that megaesophagus is a syndrome in which the responsible neuromuscular mechanism can be damaged by different agents. Some day perhaps I shall have the opportunity of visiting Brasil and if I do ... it certainly would be an exciting experience for me medically."

Em outubro de 1959 iria realizar-se em Belém, PA, o XI Congresso Brasileiro de Gastroenterologia, sendo presidente o Dr. Orlando Rodrigues da Costa e secretáriogeral o Prof. Afonso Rodrigues Filho. Naquele congresso seria discutido o relatório de um Inquérito Nacional sobre megaesôfago, do qual participaram várias sociedades estaduais, dentre as quais a Sociedade Goiana de Gastroenterologia, representada no inquérito pelo Dr. Jerônimo de Moraes Sobrinho.

Seria uma ocasião apropriada para convidar o Prof. Ingelfinger a visitar o Brasil na qualidade de conferencista estrangeiro e aproveitar a oportunidade para um contato pessoal dos médicos brasileiros interessados em fisiologia e fisiopatologia do esôfago com a maior autoridade nesta área.

Por iniciativa do Prof. Geraldo Siffert de Paula e Silva, coordenador e relator do Inquérito Nacional sobre megaesôfago, o convite foi feito oficialmente pela Federação Brasileira de Gastroenterologia e pela Sociedade Goiana de Gastroenterologia ao Prof. Ingelfinger, que aceitou vir ao Brasil como conferencista estrangeiro do referido Congresso.

Participaram do Inquérito Nacional: Ruy Ferreira Santos, de Ribeirão Preto; Nereu deAlmeida Júnior, de Belo Horizonte; Francisco Pinto de Castro, do Rio de Janeiro; Paulo Borba, de Recife; Aluízio Prata, de Salvador; Jerônimo de Moraes Sobrinho, de Goiânia; Breno Difini, de Porto Alegre, Paulo Travassos Filho, de Campos, Luiz Heraldo Câmara-Lopes dos Anjos (secretário ad hoc), de Ribeirão Preto, e como convidado especial o Prof. Franz J. Ingelfinger. Também tomaram parte nas discussões preparatórias do relatório final o Dr. Júlio Croce, de São Paulo, e Danilo Perestrello, psiquiatra, do Rio de Janeiro.

O Prof. Ingelfinger, que acompanhou a exposição e os debates dos participantes, ao término dos trabalhos, emitiu a seguinte declaração:

"A medical meeting at which the participants can discuss a combined clinical experience with some 3.000 clinical cases of esophageal aperistalsis is phenomenal in itself. Even more important, however, is that some of these cases have been studied with care and imagination so that the nature and course of aperistalsis could be discussed at the Inquiry on the basis of an unsurpassed quantity and quality of information. This information, the spirited discussion it engendered and the conclusions that it permitted convince me that Brazilian medicine is far ahead of rest of the world in understanting aperistalsis of the esophagtus and in discovering its cause".

Ficara acertado que, após o Congresso em Belém, o Prof. Ingelfinger viajaria diretamente a Goiânia, acompanhado pelo Prof. Câmara-Lopes, seguindo de Goiânia para Ribeirão Preto e, desta cidade, para São Paulo e Rio de Janeiro, de onde retornaria aos Estados Unidos. Otrajeto programado, portanto, era exatamente o inverso do habitual em viagens de convidados estrangeiros, que normalmente desembarcam no Rio de Janeiro e São Paulo para depois se dirigirem a outras cidades brasileiras.

Em carta de 25/9/1959, um de nós (JMR) solicitou ao Prof. Ingelfinger que trouxesse consigo algumas ampolas de Mecholyl para a realização do teste por ele descrito na acalásia, dada a dificuldade em obter-se o produto no Brasil em tempo hábil.

Tudo correu como fora programado. De Belém, Ingelfinger e Câmara-Lopes viajaram em vôo direto para Goiânia no dia 10/ 10/1 959. O Prof. Geraldo Siffert não pôde acompanhá-los nessa viagem em virtude de outros compromissos assumidos, ficando de esperar o Prof. Ingelfinger no Rio de Janeiro. No dia seguinte, como era domingo, ambos, Ingelfinger e Câmara Lopes, foram visitar Brasilia, a nova capital ainda em construção, retornando à Goiânia.

Na manhã do dia 12/10/1959 realizouse em Goiânia a reunião programada para exame de casos de megaesôfago. Tinham sido agendados 20 pacientes, tendo comparecido 19. A reunião se realizou no Hos- 
pital Rassi, cujas instalações foram gentilmente colocadas à disposição do ilustre visitante pelo Dr. Anis Rassi, um dos proprietários do citado Hospital. Participaram da reunião, além de Ingelfinger e Câmara-Lopes, um dos autores (JMR), Anis Rassi e Gilvan Juvenal Dutra, radiologista do Hospital.

Ingelfinger examinou as radiografias e histórias clínicas de vários pacientes, fez inúmeras perguntas e realizou, ele mesmo, radioscopia em mais de um paciente. Finalmente foi feito 0 teste da metacolina em um dos pacientes, de nome Josafá Romão, sob controle radioscópico. Tratava-se de um paciente chagásico com megaesôfago e sem cardiopatia ao exame clínico, radiológico e eletrocardiográíco. A solução de metacolina foi injetada por via subcutânea e logo depois o paciente acusou dor retroesternal coincidindo com o aparecimento, àradioscopia, de fortes contrações da metade inferior do esôfago. Entusiasmado, Ingelfinger exclamou: "It is the same disease!".

Nesse mesmo dia, às 20h30, o Prof. Ingelfinger proferiu uma conferência na Sociedade Goiana de Gastroenterologia sobre fisiologia e fisiopatologia do esôfago, que foi traduzida pelo Prof. Câmara Lopes e pelo Dr. Milton Barbosa de Lima.

Ingelfinger era uma figura carismática. De elevada estatura, impressionava pelo seu porte (fig I). Sua fisionomia descontraída e seu olhar penetrante demonstravam interesse pelo interlocutor e irradiavam simpatia. Falava com desembaraço e expressava com clareza o seu pensamento, polarizando as atenções.

No dia 13 de outubro, à tarde, Ingelfinger viajou em companhia de CâmaraLopes para Ribeirão Preto, onde, na Faculdade de Medicina local, durante três dias, deu prosseguimento à intensiva aquisição de conhecimentos que fazia sobre a moléstia de Chagas e seus "megas". Os detalhes desta visita foram relatados por Câmara-Lopes em carta datada de 15 de novembro de 1959 e enviada a um de nós (JMR). As narrativas que

\section{Fig. I - Prof. Franz J. Ingelfinger por ocasião de sua visita a Goiânia, de 10 a I3 de outubro de 1959, ao lado do Prof. Joffre M. de Rezende.}

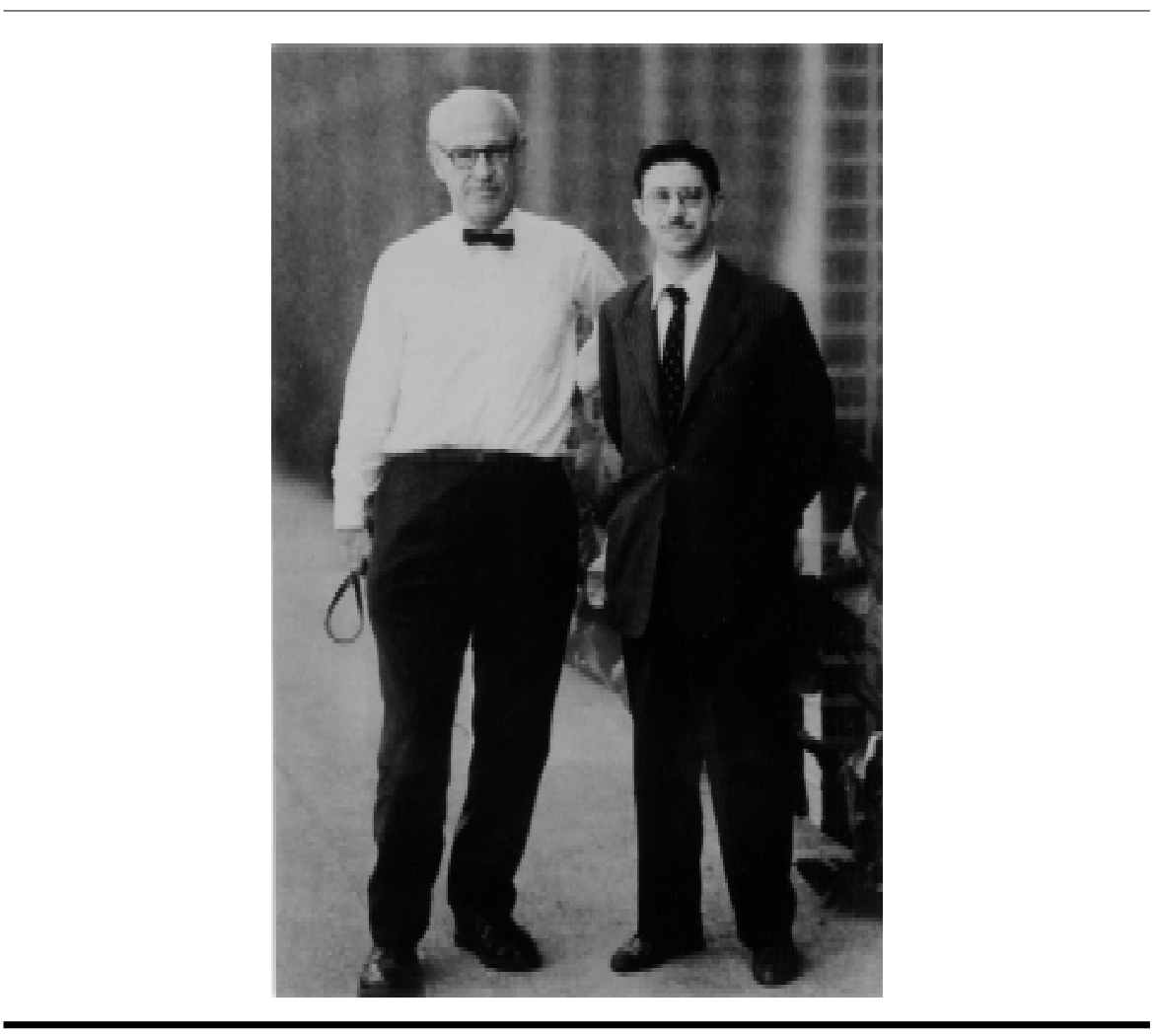

serão feitas a seguir fundamentaram-se, em grande parte, no conteúdo desta carta².

No dia seguinte à sua chegada, no Hospital das Clínicas, Ingelfinger assistiu ao segundo tempo de uma esofagectomia subtotal com esofagogastroplastia cervical transmediastinal anterior, realizada por CâmaraLopes, que a idealizara como forma de tratamento para as formas avançadas do megaesôfago chagásico. No Serviço de Radiologia, Ingelfinger acompanhou exames radioscópicos e participou de discussões sobre interpretação de radiografias do esôfago de pacientes em diferentes fases evolutivas da doença e de pacientes submetidos à esofagectomia subtotal. Ali, Ingelfinger demonstrou que, mesmo na posição de Trendelenburg, o contraste baritado contido no esôfago ou no estômago intratorácico, após esofagectomia subtotal, não refluía e nunca chegava à boca graças à ação do esfíncter esofágico superior; esta observação tranqüilizou Câmara-Lopes uma vez que se convenceu de que não haveria risco da ocorrência de faringite de refluxo como complicação da técnica operatória que preconizara.

Na manhã de 15 de outubro, Ingelfinger assistiu à ressecção de um megaesôfago chagásico e mostrou-se entusiasmado, pois nesses dias é que estava assistindo, pela primeira vez, a intervenções cirúrgicas no tórax. O megaesôfago retirado nesta ocasião apresentava enorme placa de leucoplasia na mucosa de seu terço superior. 0 órgão retirado foi levado por ele para seu hospital em Boston e, conforme informação pessoal do Prof. Luiz de Paula Castro, Ingelfinger disse-lhe, pouco depois de seu regresso, que tivera grandes dificuldades na alfândega americana para que a peça pudes- 
se entrar nos Estados Unidos. Ingelfinger encaminhou-a para o museu de anatomia patológica de seu hospital.

Além de ver casos de esofagopatia chagásica, desde incipientes até com graus avançados da doença, Ingelfinger teve informações sobre a epidemiologia e a profilaxia da doença de Chagas com os Professores Pedreira de Freitas e Nagib Haddad e, no Departamento de Parasitologia, o Prof. Astolfo Siqueira mostrou-lhe exemplares de triatomíneos transmissores do Trypanosoma cruzi, criados em laboratório.

Como não poderia deixar de fazê-lo, Ingelfinger visitou o Departamento de Patologia. Como, nesta data, o Prof. Fritz Koeberle estava ausente da cidade, foi recebido pelo Dr. Godofredo Koeberle e pelo Prof. Paulo Becker. Na sala de autópsia foram mostrados a Ingelfinger inúmeros corações, megaesôfagos e megacólons chagásicos conservados em formol. A seguir, inteirouse dos estudos quantitativos sobre a população de neurônios intramurais do tubo digestivo de pacientes com comprometimento digestivo pela moléstia de Chagas, comparativamente à de não-chagásicos. A expressiva redução ou a quase extinção dos neurônios intramurais observada em casos de megaesôfago e de megacólon muito impressionou Ingelfinger, tanto que incluiu matéria a respeito" no The Year Book of Medicine, do qual era um dos editores.

Na visita ao Departamento de Clínica Médica, os Professores Clóvis Bühler Vieira, Renato Alves de Godoy e Hélio Lourenço de Oliveira transmitiram a Ingelfinger os primeiros resultados dos estudos que se iniciavam sobre a fisiopatologia da forma digestiva da doença de Chagas.

Um dos pontos de realce da visita de Ingelfinger ocorreu no dia 16 de outubro. Na época, Godoye Vieira iniciavam estudos sobre a deglutição de pacientes chagásicos utilizando-se do método manométrico. Pretendiam, também, mediante a adminis-

Fig. 2 - Primeiro registro gráfico da hiper-reatividade motora do corpo do esôfago ao Mecholyl em paciente com megaesôfago chagásico, realizado no Hospital das Clínicas da Faculdade de Medicina de Ribeirão Preto aos 16 de outubro de 1959. Foi utilizado sistema de dois balóes de látex posicionados em diferentes alturas do corpo do esôfago e registro em quimógrafo. Inicialmente, foram feitos os dois registros apresentados na parte inferior da figura: após período de ajustes, traçam-se apenas movimentos respiratórios e onda de contração não-peristáltica. Na parte superior da figura, nota-se que em ambos os canais de registro, decorridos cerca de cinco minutos após aplicação de $3,0 \mathrm{mg}$ da droga, ocorreu aumento da atividade tônica (elevação da linha de base do traçado) e fásica (contrações amplas e freqüentes) do órgão (teste positivo), indicativos de sua desnervação.

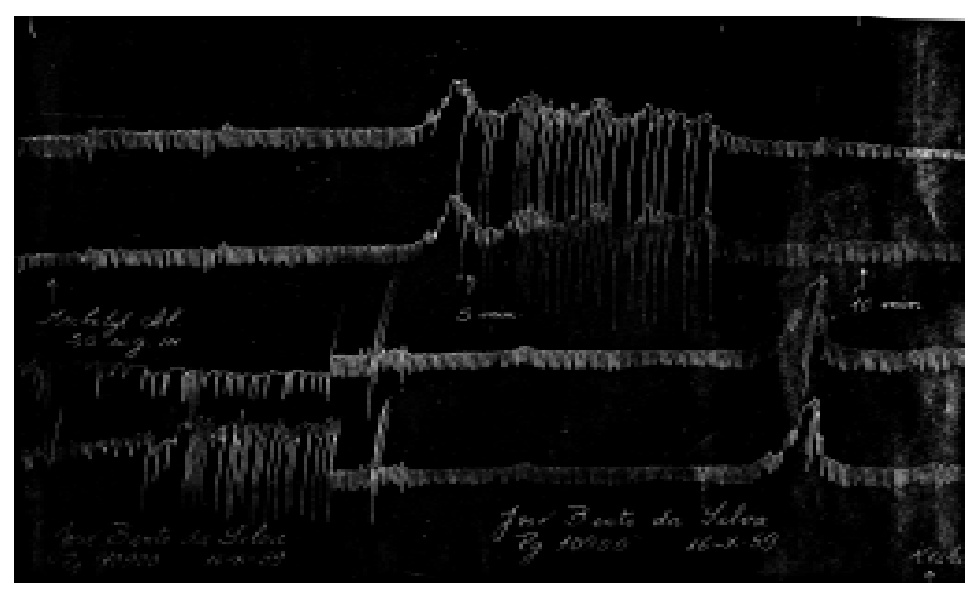

tração de uma droga colinérgica, como teste clínico, demonstrar a presença de desnervação intramural, como Ingelfinger já o fizera com o Mecholyl (metacolina - análogo da acetilcolina) na acalásia idiopática ${ }^{10,19,20}$ e já ensaiavam alguns testes com a pilocarpina. Nesse dia, com a participação de Ingelfinger e utilizando a droga que trouxera consigo, realizou-se o primeiro teste do Mecholyl com resposta motora analisada por manometria. O paciente era um chagásico (JBS - Registro HCFMRP 10.900) portador de megaesôfago. Utilizou-se o método quimográfico clássico: dois pequenos balões de látex montados em suas respectivas sondas, parcialmente preenchidos com ar, foram posicionados no lume do esôfago, um na parte média, outro na parte inferior do órgão; as sondas foram ligadas a tambores de Marey conectados a penas especiais que inscreviam em papel enfumaçado disposto em um cilindro giratório. Após um teste preliminar com pilocarpina, foram realizados dois testes sucessivos, respectivamente, com 1,5 e 3,0 mg de Mecholyl, por via intramuscular, intervalados por tempo apropriado; a dose maior provocou aumento das atividades tônica e fásica do órgão (fig 2); como, nessa dose, o esôfago normal não mostra qualquer reação, concluiu-se que a resposta motora observada no megaesôfago chagásico era hiper-reativa e, interpretada à luz da lei da desnervação de Cannon, ${ }^{3}$ demonstrava que a estrutura muscular da víscera estudada estava desprovida de inervação colinérgica.

Oito meses depois, em 17 de junho de 1960, Godoy e Vieira apresentaram à Sociedade de Biologia de Ribeirão Preto seus estudos, que demonstraram em 14 cha- 
gásicos a hiper-reatividade a drogas colinérgicas no esôfago dilatado e na doença incipiente, ainda sem dilatação do órgão;? esta última verificação era demonstrativa de que a desnervação precedia a dilatação da víscera. Posteriormente, o teste do Mecholyl foi utilizado como importante prova da condição de desnervação de outros segmentos do tubo digestivo ${ }^{22,25}$ e dos brônquios de chagásicos ${ }^{8}$.

A figura 3 mostra Ingelfinger ao lado dos professores que o recepcionaram na Faculdade de Medicina de Ribeirão Preto fotografados em corredor do antigo Hospital das Clínicas, hoje Unidade de Emergência.

Prof. Ingelfinger ficou deveras impressionado com a elevada prevalência do megaesôfago no Brasil Central. Além dos pacientes agendados, teve oportunidade de conhecer outras pessoas acometidas do mal. Em entrevista concedidaà TV-Tupi, no Rio de Janeiro, noticiada pela Tribuna Médica de I I/I I/ /959, Ingelfinger declarou que "em Goiânia lhe foram mostrados num só dia um número de casos clínicos maior do que ele já tinha visto em toda a sua vida"29.

Em carta de 2/II/1959, endereçada a um de nós (JMR), após o seu regresso aos Estados Unidos, fez o seguinte comentário:

"I shall never forget the profesional aspects of my visit to your pleasent city, for it was here that I first realized the magnitude of the problem of esophageal aperistalsis in your part of the world. Of course, I had heard about e read about it, and this was one of the reasons I was coming to Goiania. Nevertheless, the actual experience far exceed my anticipation"

Os desdobramentos que se seguiram a esta visita do Prof. Ingelfinger ao Brasil tiveram efeito favorável no sentido de difundir no exterior e dar maior credibilidade à etiologia chagásica do megaesôfago e megacólon endêmicos e à existência real de uma forma digestiva da doença de Chagas, decorrente de lesões degenerativas do siste-

\section{Fig. 3 - Prof. Franz J. Ingelfinger em Ribeirão Preto. Da esquerda para a direita, na foto: Dr. Wilson Menegucci, Prof. Renato Alves de Godoy, Prof. L.H. Câmara-Lopes, Prof. Ruy Ferreira-Santos, Prof. Franz J. Ingelfinger, Prof. J.L. Pedreira de Freitas e Prof. Clóvis Bühler Vieira.}

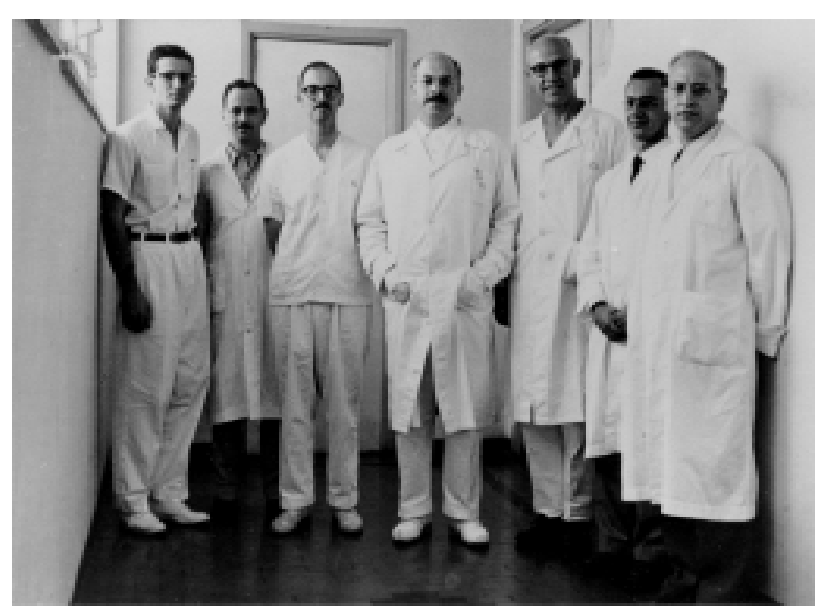

ma nervoso entérico, conforme os trabaIhos de Koeberle16-18. Esta nova visão etiopatogênica da doença de Chagas não era bem aceita, até então, nos meios científicos do Rio de Janeiro e São Paulo ${ }^{28}$.

Otrabalho publicado por um de nós(IMR) em 1959, sob o título de "Forma digestiva da Doença de Chagas", ${ }^{27}$ foi resumido e comentado por Ingelfinger no Year Book of Medicine de |960-196|'12. Como editor da seção de gastroenterologia, Ingelfinger era conhecido por sua criteriosa seleção dos trabalhos e por seus judiciosos comentários ${ }^{5}$.

Por ocasião do VI Congresso Panamericano de Gastroenterologia, que se realizaria em New York em abril de 1962, Koeberle, Câmara-Lopes e um de nós (JMR) recebemos convite do Prof. Ingelfinger para ir a Boston visitar o seu serviço no Evans Memorial Hospital e ali participar de uma Informal discussion sobre doença de Chagas e sistema digestivo.

Coincidentemente, o dia da visita a Boston, 18 de abril, eraferiado local. Apesar disso, o anfiteatro estava repleto e os três convidados apresentaram, cada um por sua vez, para uma platéia interessada e atenta, os principais aspectos clínicos, anatomopatológicos e cirúrgicos relativos ao megaesôfago chagásico e à doença de Chagas de uma maneira geral. Seguiu-se um debate com várias perguntas por parte dos presentes.

Durante a visita ao serviço do Prof. Ingelfinger no Hospital, era patente o interesse do staff pela doença de Chagas e suas manifestações digestivas.

De 13 a 19 de maio de 1962 realizouse em Munich, na Alemanha, sob a presidência do Prof. Norbert Henning, o II Congresso Mundial de Gastroenterologia. Henning havia estado no Brasil em 1961, inclusive em Goiânia, conhecia os trabalhos de Koeberle e estava bem informado sobre a alta prevalência do megaesôfago em certas regiões do Brasil e sua associação com a doença de Chagas.

Fazia parte do programa do Congresso um Simpósio (Panel discussion) sobre acalásia do esôfago, com a duração de qutro horas, sendo presidente o Prof. Franz Ingelfinger e moderador o Prof. Geraldo Siffert de Paula e Silva. Tomaram parte neste Simpósio 
como convidados: G.R.A. Vantrappen, da Bélgica; S. Kawashima, do Japão; G. McHardy e E.C. Texter, dos Estados Unidos; J. R. Trounce, da Inglaterra; Ch. Debray, da França; J.M.de Rezende, F. Koeberle e L.H. Câmara-Lopes, do Brasil (fig. 4).

A superioridade numérica dos convidados brasileiros neste Simpósio devia-se, em parte, ao prestígio pessoal do Prof. Geraldo Siffert, e em parte, ao interesse despertado pela doença de Chagas como causa importante de uma modalidade de acalásia.

O Prof. Koeberle fez sua apresentação de improviso, em alemão, tendo sido aplaudido pela assistência de pé, fato incomum em um Congresso de tal magnitude. Em seus comentários finais, assim se expressou o Prof. Ingelfinger:

"It is not accidental that four members of this panel hail from Brazil, for in this country the esophageal disease we are discussing is prevalent to a degree unheard of in other areas of the world represented at this Congress". E, mais adiante: "It thus seems to me that we should accept the Brazilian form of achalasia as a model pointing out to us a possible way in which our own cases may develop. Perhaps ours cases too are the late results of an infection or infestation which damages Auerbach's plexus by a toxic or possibly autoimmune mechanism and then subsides, leaving no traces at the time when the late effects of the neurologic damage finally make themselves manifest in the form of achalasia. The Brazilians have given us a major lead"13.

Sabe-se que a importância da doença de Chagas para a saúde pública restringe-se, geograficamente, aos países sul-americanos, particularmente ao Brasil. Ingelfinger, entretanto, percebeu que "the Brazilian form of achalasia" era algo mais, com um significado médico muito mais amplo, universal. Entendeu ele que a doença brasileira constituía-se em um modelo patológico peculiar em virtude da sua característica

Fig. 4 - Simpósio sobre acalásia do esôfago no II Congresso Mundial de Gastroenterologia, realizado em Munich, Alemanha, de 13 a 19 de maio de 1962. Da esquerda para a direita: J. R. Trounce, da Inglaterra; G. R. Vantrappen, da Bélgica; E.C. Texter, dos Estados Unidos; Ch. Debray, da França; F.J. Ingelfinger, dos Estados Unidos, presidente da sessão; Geraldo Siffert, do Brasil, moderador; G. Mc Hardy, dos Estados Unidos; L.H. Câmara-Lopes, Joffre M. de Rezende e Fritz Koeberle, do Brasil, e S. Kawashima, do Japão.

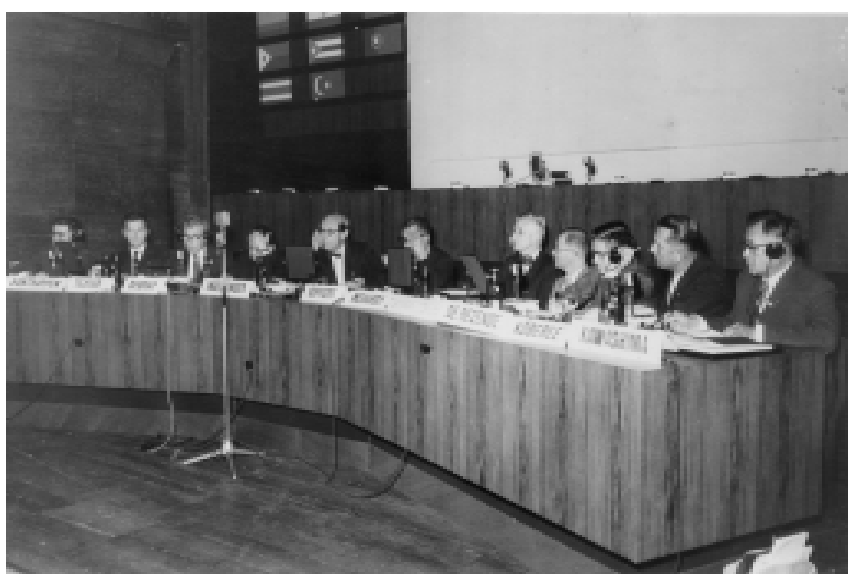

histopatológica básica - a desnervação do sistema nervoso entérico - do qual poderse-iam tirar ilações sobre a etiopatogenia, a fisiopatologia e o tratamento de doenças que the eram semelhantes. Estendendose este conceito a todos os segmentos do tubo digestivo comprometidos pela doença de Chagas, pode-se dizer que o estudo da forma digestiva da moléstia de Chagas, principalmente em seus aspectos motores, pode trazer subsídios para o entendimento de outras doenças, orgânicas e funcionais, nas quais o sistema nervoso entérico esteja envolvido em sua fisiopatologia e, além disso, pode permitir deduções sobre a participação da regulação nervosa intrínseca no comportamento fisiológico do órgão considerado. ${ }^{22,25}$ Assim, Ingelfinger foi, para o mundo, um importante arauto desta maneira de ver a forma digestiva da moléstia de Chagas.

Nota - Toda a correspondência do Prof. Franz J. Ingelfinger citada neste artigo encontra-se arquivada, em poder de um dos autores (JMR).

\section{RefERÊNCIAS}

I. Amorim M, Correia Neto A. Histopathologia e pathogenese do megaesôfago e megareto. Considerações em torno de um caso de "mal do engasgo". Ann Fac. Med. Univ. São Paulo 8: $101-127,1932$

2. Câmara-Lopes LH. Carta pessoal datada de 15 de novembro de 1959 e enviada a JM Rezende a propósito da visita de F.J. Ingelfinger a Ribeirão Preto. Cópia arquivada no Centro de Documentação e Biblioteca de História da Medicina da FAEPA, Hospital das Clínicas da Faculdade de Medicina de Ribeirão Preto (USP)

3. Cannon WB. A law of denervation. Am J Med Sci 1939; 198:737-50.

4. Correia Neto A, Etzel E. Le megaesophage et le megacolon devant la theorie de l'achalasie. Étude clinique et anatomo-pathologique. Rev Sud Americaine Med Chir 7: 395-420, 1934

5. Editorials. Franz J. Ingelfinger: his accomplishments. Gastroenterology 1981; 80 |059-7|.

6. Etzel E. Neuropatologia do megaesôfago e megacolo. Estudo de 5 casos Ann Fac Med Univ São Paulo 1934; 10:383-95.

7. Freitas JLP. Contribuição para o estudo do diagnóstico da moléstia de Chagas por processos de laboratório [tese]. São Paulo: Faculdade de Medicina da Universidade de São Paulo, 1947.

8. Godoy RA. Hypersensitivity of the bronchial muscle to Mecholyl in the chronic Chagas disease. Rev Goiana Med 1964; 10: 91 - I 19.

9. Godoy RA. Vieira CB. Effects of cholinergic drugs on the esophagus of patients with Chagas disease. Acta Physiol Latino Am 196 I; I I: 107 
10. Ingelfinger FJ. Cardiospasm. Bull New Engl Med Center 1948: 10: 25।-4.

II. Ingelfinger FJ editor. Chagas' megaesophagus: quantitative study of Auerbach's plexus. The Year Book of Medicine, 19601961. Series, part 5: The Digestive System. p. 469-470.

12. Ingelfinger FJ editor. Digestive form of Chagas' disease. The Year Book of Medicine, 1960-1961. Series, part 5: The Digestive System. p. 466-9.

13. Ingelfinger FJ. An 2nd World Congress of Gastroenterologie. V I. Topical problems in achalasia of the esophagus, Basel: S.Karger, 1963; p. 44-45

14. Ingelfinger FJ. Esophageal motility. Physiol Reviews 1958; 38:533-84.

15. Koeberle F, Nador E. Etiologia e patogenia do megaesôfago no Brasil. Rev Paul Med 1955; 47:643-61

16. Koeberle F-Chagaskrankheit: Eine Erkrankung der neurovegetativen Peripherie. Wien Klin Wchnschr 1956; 68:333-39.

17. Koeberle F. Patogenia da moléstia de Cha- gas. Estudos dos órgãos musculares ocos. Rev Goiana Med 1957; 3: I55-80.

18. Koeberle F. Moléstia de Chagas. Enfermidade do sistema nervoso autônomo. Anais Congresso Internacional Doença de Chagas 1959, |961;69|-7|6.

19. Kramer P, Ingelfinger FJ. Cardiospasm, a generalized disorder of esophageal motility. Am J Med 1949; 7: 174-9.

20. Kramer $P$, Ingelfinger FJ. Esophageal sensitivity to Mecholyl in cardiospasm. Gastroenterology 1951; 19:242-53.

21. Laranja FS, Dias E, Nobrega G. Estudo eletrocardiográfico de 81 casos de megaesôfago. Mem Inst Oswaldo Cruz 1948; 46: 473-29.

22. Meneghelli UG. Chagas' disease: a model of denervation in the study of digestive tract motility. Braz J Med Biol Res 1985; I 8: 255-64.

23. Mineiro V. Contribuição à etiologia do megaesôfago. Distribuição geografia. Comunicação ao $2^{\circ}$ Congresso Médico do Triângulo Mineiro e $1^{\circ}$ do Brasil Central realizado em Uberlândia em 1948. Rev Goiana Med 1958; 4:29-34.
24. Okumura M, Correia Neto A. Etiopatogenia do megacolo chagasico. Contribuição experimental. Rev Hosp Clin Fac Med São Paulo 1963; 18:351-60.

25. Oliveira RB, Troncon L, Dantas RO, Meneghelli UG. Gastrointestinal manifestations of Chagas' disease. Am J Gastroenterol 1998; 93: 884-89.

26. Rezende JM. Megaesôfago por doença de Chagas. Rev Goiana Med 1956; 2:297-3। 4.

27. Rezende JM. Forma digestiva da moléstia de Chagas. Rev Goiana Med 1959; 5: 193-227.

28. Rezende JM. Megaesôfago chagásico (mal de engasgo). Dados históricos sobre o reconhecimento de sua etiologia. Gastroenterol Contemp 1998; 2:6-15.

29. Tribuna Médica. Rio de Janeiro, II nov. de 1959

Artigo recebido: 25/09/2000 Aceito para publicação: 19/01/2001

\section{OPINIÃODOLEITOR}

A nova Ramb abre espaço para o leitor na seção "Correspondências".

Envie sua opinião ou sugestão sobre a revista ou artigos

de seu interesse. Participe também enviando suas dúvidas ou sugestões sobre condutas práticas para a seção "À beira do leito".

Sua colaboração é muito importante para aprimorarmos o nosso

veículo de comunicação. Rua São Carlos do Pinhal, 324

Cep:01333-903-SãoPaulo-SP-E-mail:ramb@amb.org.br 